\title{
ANZTLA 2012 Statistics
}

by Kerrie Stevens

ANZTLA Statistician

NZTLA Statistics are compiled annually from data provided by ANZTLA member
libraries on a voluntary basis.

The reporting categories are described as follows:

- A Provides clergy training programmes (ie: The Australian and New Zealand Association of Theological Schools [ANZATS] member schools) and/or schools and training institutions which offer broad theological training (ie: Bible Colleges, Missionary Training Institutions) possibly leading to degree or post-graduate qualifications;

- B Do not have students (ie: church administrative libraries, resource centres, parachurch organizations, etc.); and

- C Institutions offering non-theological courses in addition (ie: teacher training).

Data has been entered as submitted by the libraries. Any questions should be directed to the libraries concerned.

Thank you to all libraries who contribute to the ANZTLA Statistics! 
TABLE 12012 UBRARY INFORMATION

\begin{tabular}{|c|c|c|c|c|}
\hline 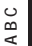 & INSTITUTION & UBRARY NAME & STATENZ & PREDOMNANT DENOMNATON OF COUECTION \\
\hline A & Adelaide College of Divinity & Adelaide Theological Library & SA & Well-spread over all denominations \\
\hline c & Avondale College of Higher Education & Avondale Libraries (Lake Macquarie Campus only) & NSW & Seventh Day Adventist \\
\hline c & Australian Catholic University & Australian Catholic University Library (6 campus & & Catholic \\
\hline A & Australian Lutheran College & Löhe Memorial Library & SA & Lutheran \\
\hline A & Benedictine Community of New Norcia & Library & WA & Catholic \\
\hline c & Bethlehem Tertiary Institute & Library & $\mathrm{NZ}$ & \\
\hline A & Bible College of South Australia & Burrow Library & SA & Anglican, Protestant, Methodist, Baptist \\
\hline $\mathrm{A}$ & Carey Baptist College & Ayson Clifford Library & NZ & Baptist, Protestant \\
\hline A & Catholic Diocese of Auckland & Catholic Diocese of Auckland Library & NZ & Catholic \\
\hline $\mathrm{B}$ & Catholic Education Office & TCEO iLibrary & QLD & Catholic \\
\hline \multirow{2}{*}{$B$} & Catholic Education Office & Catholic Resource \& Information Service & SA & Catholic \\
\hline & Catholic Education Office & Catholic Library of Western Australia & WA & Catholic \\
\hline A & Catholic Institute of Sydney & Veech Library & NSW & Catholic \\
\hline A & Catholic Theological College & Daniel Mannix Library & $\mathrm{VIC}$ & Catholic \\
\hline \multirow{2}{*}{ A } & Crossway College & W.J. Tunley Memorial Library & QLD & Well-spread over all denominations \\
\hline & Eastwest College of Intercultural Studies & Chee Memorial Library & NZ & Interdenominational missions \\
\hline A & Emmaus Bible College & Emmaus Bible College Library & NSW & Brethren Movement, Well-spread over all \\
\hline A & Franciscan Order of Friars Minor & St Paschal Library & $\mathrm{VIC}$ & Catholic \\
\hline A & Greek Orthodox Archdiocese of Australia & St Andrew's Greek Orthodox Theological College Library & NSW & Orthodox \\
\hline A & Harvest Bible College & Harvest Bible College Queensland Library & QLD & Pentecostal, Well-spread over all denoms \\
\hline A & Harvest Bible College & Harvest Bible College Main Library & $\mathrm{VIC}$ & Pentecostal, Well-spread over all denoms \\
\hline A & Laidlaw College & Deane Memorial Library & NZ & Well-spread over all denominations \\
\hline \multirow{2}{*}{$\mathrm{A}$} & Malyon College, Queensland Baptist College of & Malyon College Library & QLD & Baptist \\
\hline & Mary Andrews College & Library & NSW & Anglican \\
\hline \multirow[t]{2}{*}{ A } & Melbourne School of Theology & J.W. Searle Library & $\mathrm{VIC}$ & Well-spread over all denominations \\
\hline & Nazarene Theological College & John D. Fulton Library & QLD & Methodist \\
\hline A & Perth Bible College & Urquhart Memorial Library & WA & Well-spread over all denominations \\
\hline A & Presbyterian Research Centre & Hewitson Library & $\mathrm{NZ}$ & Well-spread over all denominations \\
\hline A & Presbyterian Theological Centre & Presbyterian Theological Centre Library & NSW & Presbyterian, Reformed \\
\hline A & Queensland Theological College & Gibson-Radcliffe Library & QLD & Presbyterian \\
\hline \multirow[t]{2}{*}{$\mathrm{A}$} & Ridley Melbourne Mission \& Ministry College & Leon Morris Library & $\mathrm{VIC}$ & Anglican \\
\hline & Salvation Army & Booth College of Mission & $\mathrm{NZ}$ & Salvation Army \\
\hline A & St Athanasius Coptic Orthodox Theological College & St Athansius Coptic Orthodox Library & VIC & Coptic Orthodox \\
\hline$B$ & St Benedict's Monastery & St Benedict's Monastery Library & NSW & Catholic \\
\hline \multirow[t]{2}{*}{ A } & St Francis Theological College & Roscoe Library & QLD & Anglican \\
\hline & St Mark's National Theological Centre & St Mark's Anglican National Memorial Library & ACT & Anglican \\
\hline A & Sydney Missionary \& Bible College & Kerr Library & NSW & Protestant, Well-spread over all denoms \\
\hline \multirow[t]{2}{*}{$\mathrm{C}$} & Tabor College & Tabor College NSW Library & NSW & Well-spread over all denominations \\
\hline & Taboc College Tasmania & Library & TAS & \\
\hline C & Tabor Victoria & Library & $\mathrm{VIC}$ & Well-spread over all denominations \\
\hline A & Trinity Theological College & Trinity Theological Library & QLD & Protestant \\
\hline A & Trinity Theological College & Trinity Theological College Library & WA & Well-spread over all denominations \\
\hline A & Uniting Church in Australia & Camden Theological Library & NSW & Protestant \\
\hline A & Vianney College & St Ann's Library & NSW & Catholic \\
\hline A & Vose Seminary & Vose Library & WA & Baptist \\
\hline C & Wesley institute & Gordon Moyes Library & NZ & Well-spread over all denominations \\
\hline A & Whitley College & Geoffrey Blackburn Library & $\mathrm{VIC}$ & Baptist \\
\hline A & Youthworks College & Youthworks College Library & NSW & Anglican, Protestant \\
\hline
\end{tabular}

\begin{tabular}{|l|c|}
\hline Anglican & 6 \\
\hline Baptist & 5 \\
\hline Brethren Movement & 1 \\
\hline Catholic & 11 \\
\hline Coptic Orthodox & 2 \\
\hline Lutheran & 1 \\
\hline Methodist & 2 \\
\hline Not stated/Other & 3 \\
\hline Pentecostal & 2 \\
\hline Presbyterian & 2 \\
\hline Protestant & 6 \\
\hline Salvation Army & 1 \\
\hline Seventh Day Adventist & 1 \\
\hline Well-spread over all denominations & 14 \\
\hline
\end{tabular}

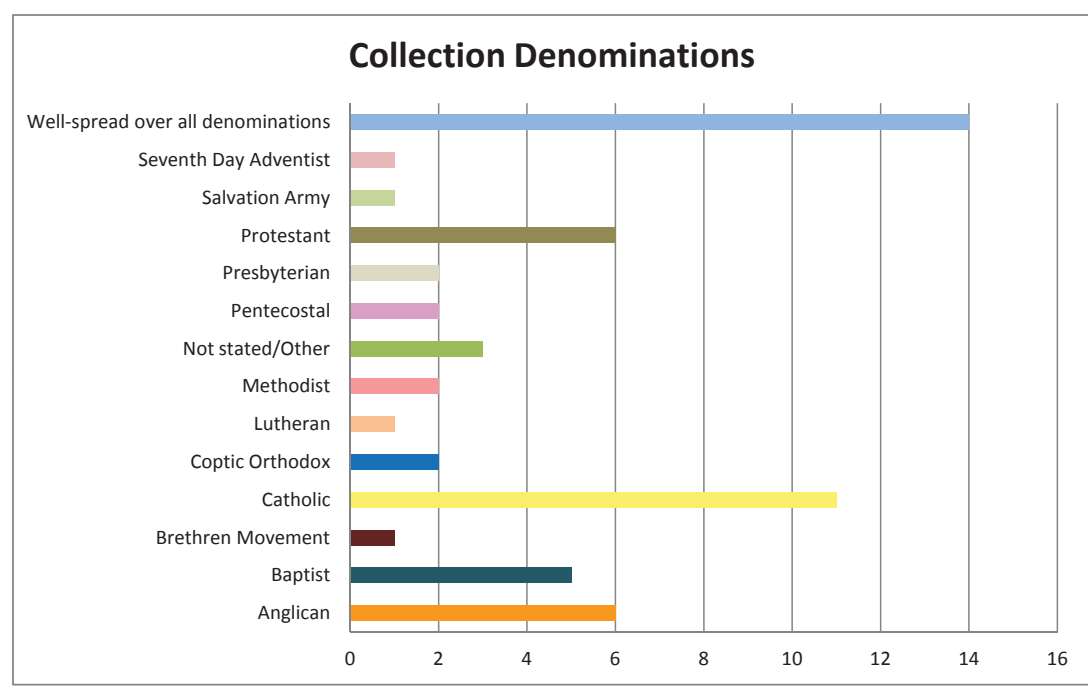


TABLE2 2012 UBRARY STAFF

\begin{tabular}{|c|c|c|c|c|c|c|c|c|}
\hline \multirow[t]{2}{*}{$\begin{array}{l}0 \\
m \\
\end{array}$} & \multirow[t]{2}{*}{ INSTITUTION } & \multirow[t]{2}{*}{$\begin{array}{c}\text { STATE } \\
\text { NZ }\end{array}$} & $\begin{array}{c}\text { PROF. } \\
\text { POSITIONS }\end{array}$ & $\begin{array}{l}\text { PARA-PROF. } \\
\text { POSITIONS }\end{array}$ & $\begin{array}{l}\text { SUPPORT } \\
\text { STAF } \\
\text { POSITIONS }\end{array}$ & VOLUNTEERS & TOTAL STAF & \\
\hline & & & {$[1]$} & [2] & [3] & [4] & [5] & \\
\hline A & Adelaide College of Divinity & SA & 1.7 & 0.5 & 0.08 & 0.15 & 2.43 & \\
\hline $\mathrm{C}$ & Avondale College of Higher Education & NSW & 4.4 & 1.6 & 0.5 & & 6.50 & \\
\hline$C$ & Australian Catholic University & & 40.1 & 34.5 & 18.6 & & 93.20 & across 6 campus libraries \\
\hline$A$ & Australian Lutheran College & SA & 3.4 & & 0.5 & 3 & 6.90 & part time EFT not given \\
\hline$A$ & Benedictine Community of New Norcia & WA & 0.6 & & & & 0.60 & \\
\hline C & Bethlehem Tertiary Institute & $\mathrm{NZ}$ & 1 & & 0.1 & & 1.10 & \\
\hline$A$ & Bible College of South Australia & SA & 0.4 & & & 0.5 & 0.90 & \\
\hline A & Carey Baptist College & NZ & 3 & & & & 3.00 & \\
\hline A & Catholic Diocese of Auckland & NZ & 0.5 & 0.5 & & 0.33 & 1.33 & \\
\hline$B$ & Catholic Education Office & QLD & 1 & & 1 & & 2.00 & \\
\hline$B$ & Catholic Education Office & SA & 1 & 1 & & 0.1 & 2.10 & \\
\hline A & Catholic Institute of Sydney & NSW & 1.5 & 1.85 & 0.5 & & 3.85 & \\
\hline A & Catholic Theological College & $\mathrm{VIC}$ & 1.8 & 1 & 0.3 & & 3.10 & \\
\hline A & Crossway College & QLD & 0.6 & & 0.1 & 0.45 & 1.15 & \\
\hline & Eastwest College of Intercultural Studies & NZ & 1 & & & 2 & 3.00 & part time EFT not given \\
\hline A & Emmaus Bible College & NSW & 0.9 & 0.2 & & 0.4 & 1.50 & \\
\hline A & Franciscan Order of Friars Minor & $\mathrm{VIC}$ & 1.8 & & 0.6 & & 2.40 & \\
\hline A & Greek Orthodox Archdiocese of Australia & NSW & & 1.4 & & & 1.40 & \\
\hline A & Harvest Bible College & QLD & 0.2 & & & & 0.20 & \\
\hline A & Harvest Bible College & VIC & 1 & & & & 1.00 & \\
\hline$A$ & Laidlaw College & NZ & 4.35 & & & 0.45 & 4.80 & \\
\hline A & Malyon College, Queensland Baptist College of & QLD & 0.86 & & & 0.26 & 1.12 & \\
\hline & Mary Andrews College & NSW & & & 0.33 & & 0.33 & \\
\hline A & Melbourne School of Theology & $\mathrm{VIC}$ & 1 & & 1 & 0.33 & 2.33 & \\
\hline A & Presbyterian Research Centre & NZ & 2 & 0.5 & & 0.5 & 3.00 & \\
\hline$A$ & Presbyterian Theological Centre & NSW & 0.9 & & 0.1 & & 1.00 & \\
\hline$A$ & Queensland Theological College & QLD & 1 & & 0.24 & 0.5 & 1.74 & \\
\hline A & Ridley Melbourne Mission \& Ministry College & VIC & 1.8 & & 0.4 & & 2.20 & \\
\hline & Salvation Army & $\mathrm{NZ}$ & 1 & & & & 1.00 & \\
\hline A & St Athanasius Coptic Orthodox Theological College & $\mathrm{VIC}$ & 0.5 & & & & 0.50 & \\
\hline$B$ & St Benedict's Monastery & NSW & 0.2 & & & & 0.20 & \\
\hline$A$ & St Francis Theological College & QLD & 1.4 & 0.35 & & 0.18 & 1.93 & \\
\hline & St Mark's National Theological Centre & ACT & 2 & 1 & 1 & 6 & 10.00 & part time EFT not given \\
\hline A & Sydney Missionary \& Bible College & NSW & 1.6 & 1 & 0.5 & 1 & 4.10 & \\
\hline C & Tabor College & NSW & 1 & 1 & & 2 & 4.00 & part time EFT not given \\
\hline & Taboc College Tasmania & TAS & 0.36 & & & & 0.36 & \\
\hline $\mathrm{C}$ & Tabor Victoria & VIC & 2 & 0.5 & & 0.6 & 3.10 & \\
\hline$A$ & Trinity Theological College & QLD & 1 & 1 & & 2 & 4.00 & part time EFT not given \\
\hline A & Trinity Theological College & WA & 1.4 & & 0.3 & 0.2 & 1.90 & \\
\hline$A$ & Uniting Church in Australia & NSW & 1 & 1.25 & 0.75 & & 3.00 & \\
\hline A & Vianney College & NSW & 0.2 & & 1 & & 1.20 & \\
\hline$A$ & Vose Seminary & WA & 0.6 & 0.6 & & 0.7 & 1.90 & \\
\hline $\mathrm{C}$ & Wesley institute & $\mathrm{NZ}$ & 1.75 & 0.75 & 0.33 & & 2.83 & \\
\hline$A$ & Whitley College & VIC & 0.8 & 0.13 & 0.2 & & 1.13 & \\
\hline $\mathrm{A}$ & Youthworks College & NSW & & & 0.2 & 0.1 & 0.30 & \\
\hline TOT & TAL & & 98.62 & 53.23 & 32.83 & 21.75 & 206.43 & \\
\hline$\overline{A V E}$ & BAGE & & 2.2413636 & 2.4195455 & 1.2626923 & 0.98863636 & 4.300625 & \\
\hline
\end{tabular}


TABLE 32012 UBRARY SERMCES

\begin{tabular}{|c|c|c|c|c|c|c|c|c|c|c|}
\hline \multirow[t]{2}{*}{$\begin{array}{l}0 \\
\infty \\
\ll\end{array}$} & \multirow[t]{2}{*}{ INSTITUTION } & \multirow[t]{2}{*}{$\begin{array}{c}\text { STATE } \\
\mathrm{NZ}\end{array}$} & $\begin{array}{c}\text { TOTAL } \\
\text { LOANS 2010 }\end{array}$ & $\begin{array}{l}\text { ORIGINAL } \\
\text { LENT }\end{array}$ & $\begin{array}{l}\text { ILLS SUPPLIED } \\
\text { COPYIFAX } \\
\text { SUPPUED }\end{array}$ & $\begin{array}{c}\text { TOTAL } \\
\text { SUPPUED }\end{array}$ & $\begin{array}{l}\text { ITEMS } \\
\text { REQ'D }\end{array}$ & $\begin{array}{c}\text { LLS REQUESTED } \\
\text { COPYIFAX } \\
\text { REQ'D }\end{array}$ & $\begin{array}{l}\text { TOTAL } \\
\text { REQ'D }\end{array}$ & \\
\hline & & & [6] & [7] & [8] & [9] & {$[10]$} & [11] & {$[12]$} & \\
\hline A & Adelaide College of Divinity & SA & 12,430 & 238 & 161 & 399 & 17 & 5 & 22 & \\
\hline $\mathrm{C}$ & Avondale College of Higher Education & NSW & 27,807 & 365 & 230 & 595 & 37 & 29 & 66 & \\
\hline $\mathrm{C}$ & Australian Catholic University & & 269,823 & 1418 & 796 & 2214 & 836 & 1011 & 1847 & across 6 campus libraries \\
\hline$A$ & Australian Lutheran College & SA & 12,607 & 50 & 50 & 100 & 15 & 26 & 41 & \\
\hline A & Benedictine Community of New Norcia & WA & 150 & & 2 & 2 & & 2 & 2 & approximation \\
\hline $\mathrm{C}$ & Bethlehem Tertiary Institute & $\mathrm{NZ}$ & 11,565 & & & & & 1 & 1 & \\
\hline$A$ & Bible College of South Australia & SA & 1,622 & & & & 2 & 3 & 5 & \\
\hline A & Carey Baptist College & $\mathrm{NZ}$ & 11,710 & 62 & 6 & 68 & 13 & 5 & 18 & \\
\hline A & Catholic Diocese of Auckland & NZ & 409 & 14 & 4 & 18 & 20 & 4 & 24 & 1 entered in error \\
\hline B & Catholic Education Office & QLD & 1,154 & & & & & & & \\
\hline $\mathrm{B}$ & Catholic Education Office & SA & 7,721 & & & & & 2 & 2 & \\
\hline & Catholic Education Office & WA & 8,865 & & & & & & & \\
\hline A & Catholic Institute of Sydney & NSW & 13,496 & & & 123 & & & 55 & \\
\hline A & Catholic Theological College & VIC & 14,813 & 30 & 11 & 41 & 33 & 13 & 46 & \\
\hline A & Crossway College & QLD & 18,391 & 4 & 7 & 11 & 6 & 22 & 28 & \\
\hline & Eastwest College of Intercultural Studies & NZ & 2,243 & & & & & & & \\
\hline A & Emmaus Bible College & NSW & 3,420 & 6 & 14 & 20 & 8 & 3 & 11 & \\
\hline A & Franciscan Order of Friars Minor & $\mathrm{VIC}$ & 7,383 & 2 & 2 & 4 & 27 & 23 & 50 & \\
\hline A & Greek Orthodox Archdiocese of Australia & NSW & 2,156 & & & & & 2 & 2 & \\
\hline A & Harvest Bible College & QLD & & & & & & & & \\
\hline A & Harvest Bible College & $\mathrm{VIC}$ & 4,860 & & & & 1 & & & \\
\hline A & Laidlaw College & NZ & 24,890 & 86 & 10 & 96 & 36 & 10 & 46 & \\
\hline A & Malyon College, Queensland Baptist College of & QLD & 5,517 & 7 & 2 & 9 & 2 & 3 & 5 & \\
\hline & Mary Andrews College & NSW & 1,580 & & & & & & & \\
\hline A & Melbourne School of Theology & $\mathrm{VIC}$ & & 3 & & 3 & 8 & 10 & 18 & \\
\hline & Nazarene Theological College & QLD & & & 2 & 2 & & & & \\
\hline A & Perth Bible College & WA & 2,565 & 1 & & 1 & & & & \\
\hline A & Presbyterian Research Centre & NZ & 5,776 & & & 12 & & & 32 & submitted in \#7 in error \\
\hline A & Presbyterian Theological Centre & NSW & 3,696 & & & & & & & \\
\hline A & Queensland Theological College & QLD & 4,402 & 1 & 1 & 2 & 28 & 26 & 54 & \\
\hline A & Ridley Melbourne Mission \& Ministry College & $\mathrm{VIC}$ & 13,738 & 3 & 4 & 7 & 1 & 4 & 5 & \\
\hline & Salvation Army & NZ & 3,864 & & & & 17 & 3 & 20 & \\
\hline A & St Athanasius Coptic Orthodox Theological College & $\mathrm{VIC}$ & & & & & & & & \\
\hline $\mathrm{B}$ & St Benedict's Monastery & NSW & & & & & 2 & & 2 & \\
\hline A & St Francis Theological College & QLD & & 2 & 5 & 7 & & & & \\
\hline & St Mark's National Theological Centre & ACT & 89,848 & 140 & & 58 & 54 & & & as submitted \\
\hline A & Sydney Missionary \& Bible College & NSW & 20,000 & 1 & 5 & 6 & 2 & 10 & 12 & \\
\hline $\mathrm{C}$ & Tabor College & NSW & 6,000 & 20 & & & 20 & & 30 & as submitted \\
\hline & Taboc College Tasmania & TAS & & & & & & & & \\
\hline C & Tabor Victoria & $\mathrm{VIC}$ & 5,284 & & & & & & & \\
\hline A & Trinity Theological College & QLD & 2,927 & 9 & 9 & 18 & 22 & 3 & 25 & \\
\hline A & Trinity Theological College & WA & & 6 & 10 & 16 & 3 & 2 & 5 & \\
\hline A & Uniting Church in Australia & NSW & 8,000 & 13 & 7 & 20 & 20 & 1 & 21 & approximation \\
\hline A & Vianney College & NSW & 1,249 & 1 & & 1 & 4 & & 4 & \\
\hline $\mathrm{A}$ & Vose Seminary & WA & 9,570 & 5 & 3 & 8 & 5 & 3 & 8 & \\
\hline C & Wesley institute & $\mathrm{NZ}$ & & & & & & & & \\
\hline$A$ & Whitley College & $\mathrm{VIC}$ & 3,021 & & 5 & 5 & 1 & & 1 & \\
\hline A & Youthworks College & NSW & 447 & & & & & & & \\
\hline TO1 & $\Gamma A L$ & & 644,999 & 2,487 & 1,346 & 3,866 & 1,240 & 1,226 & 2,508 & \\
\hline$\overline{\mathrm{AVE}}$ & EAGE & & 16,538 & 99 & 59 & 133 & 44 & 47 & 78 & \\
\hline
\end{tabular}




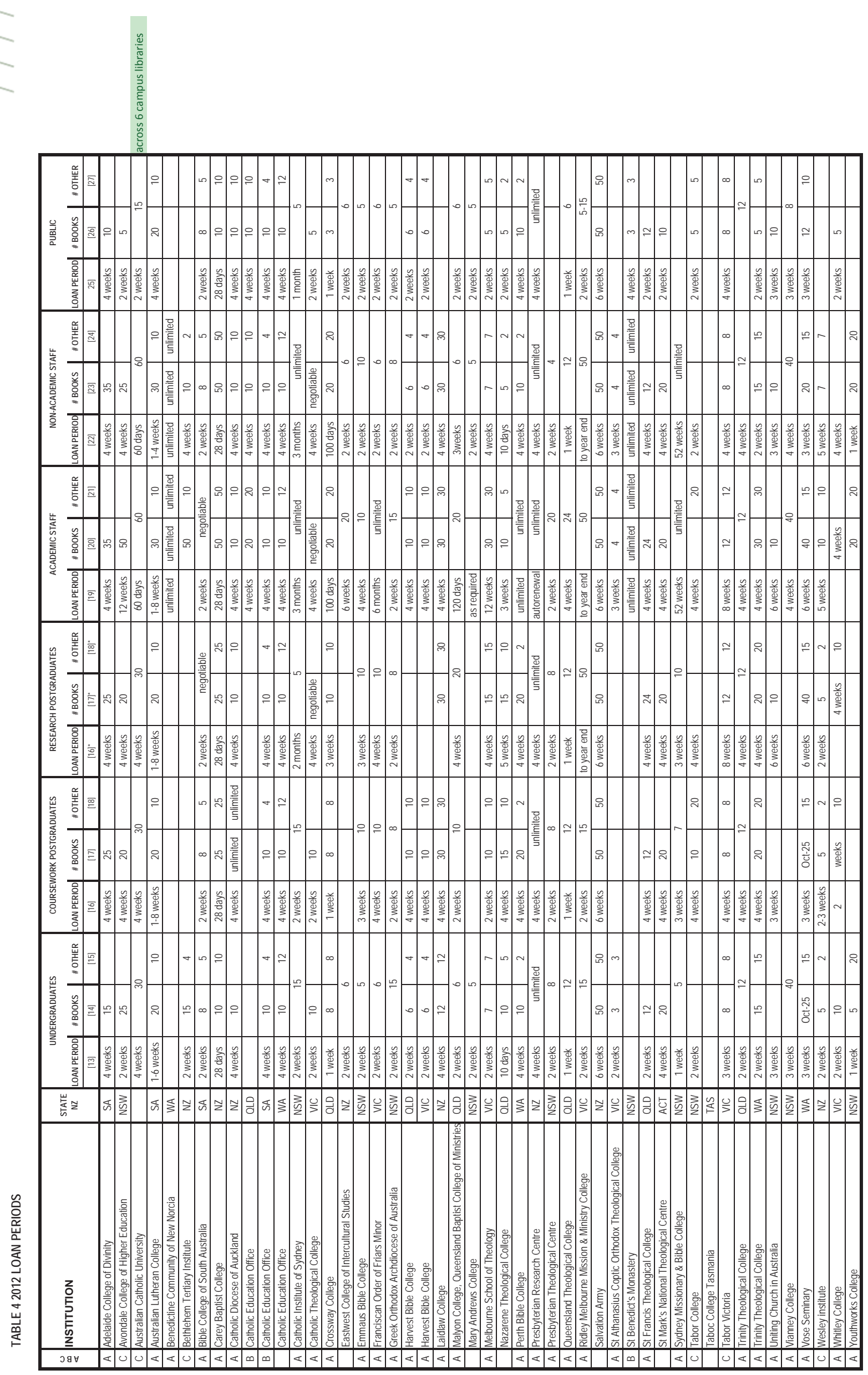




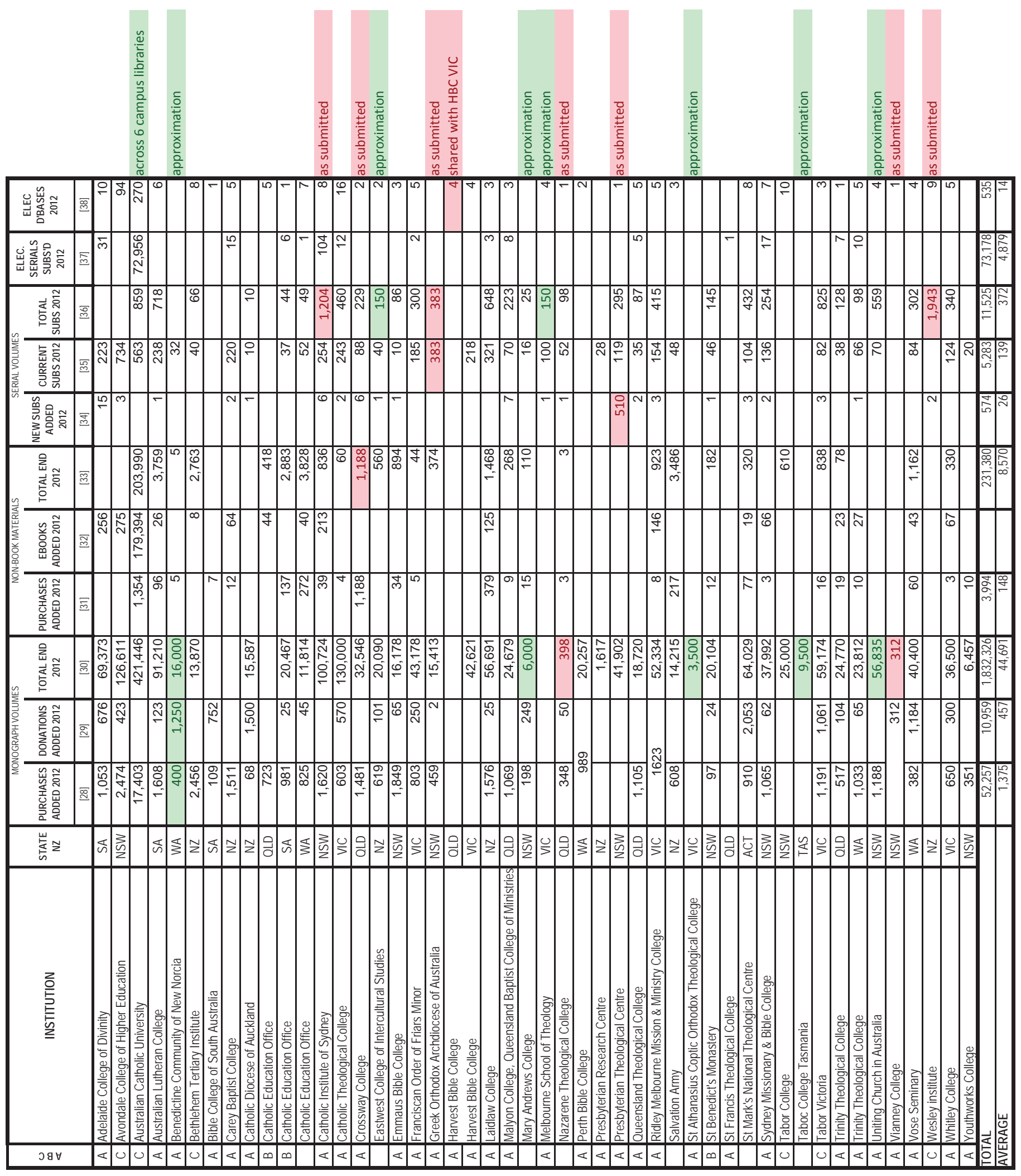




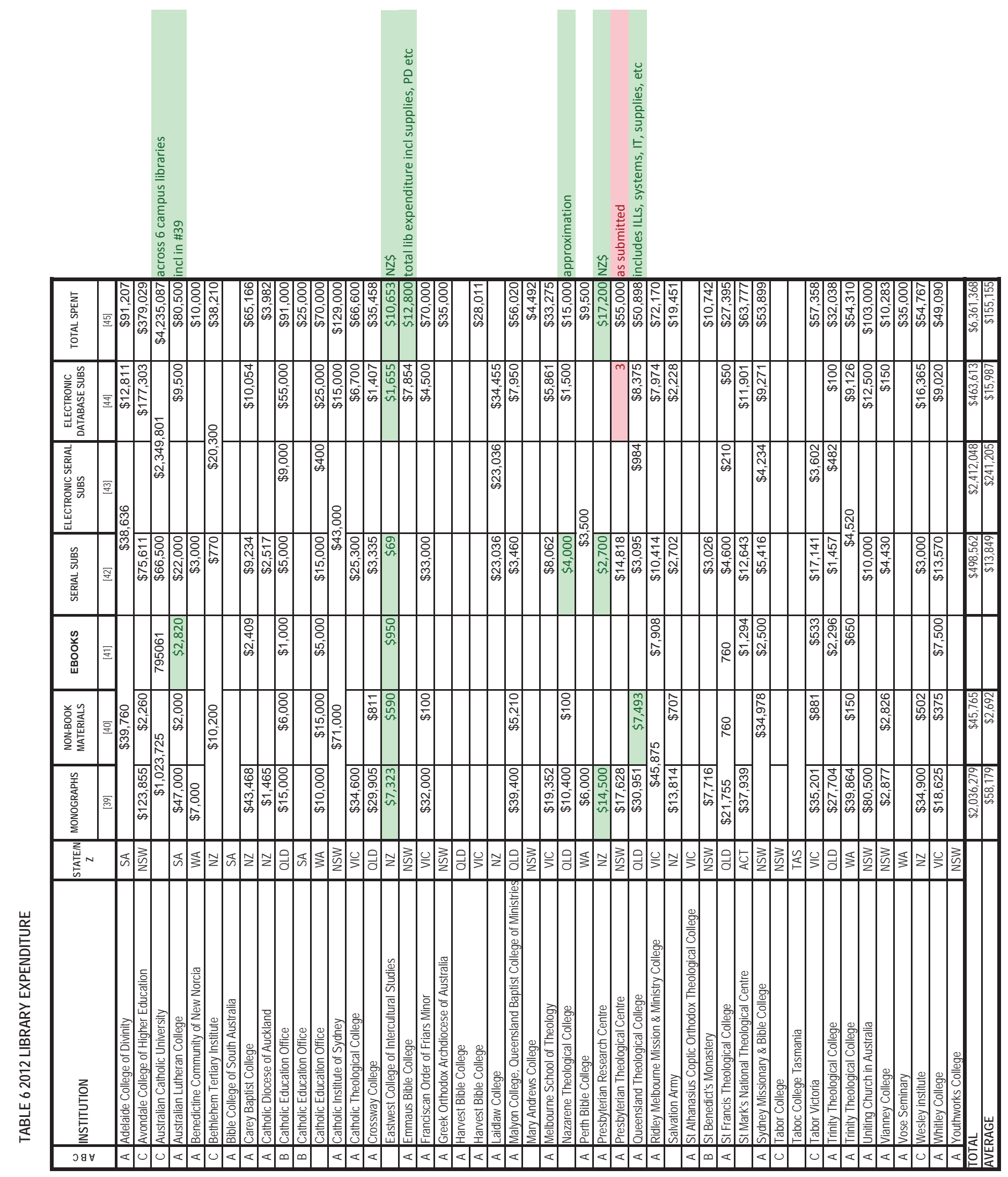




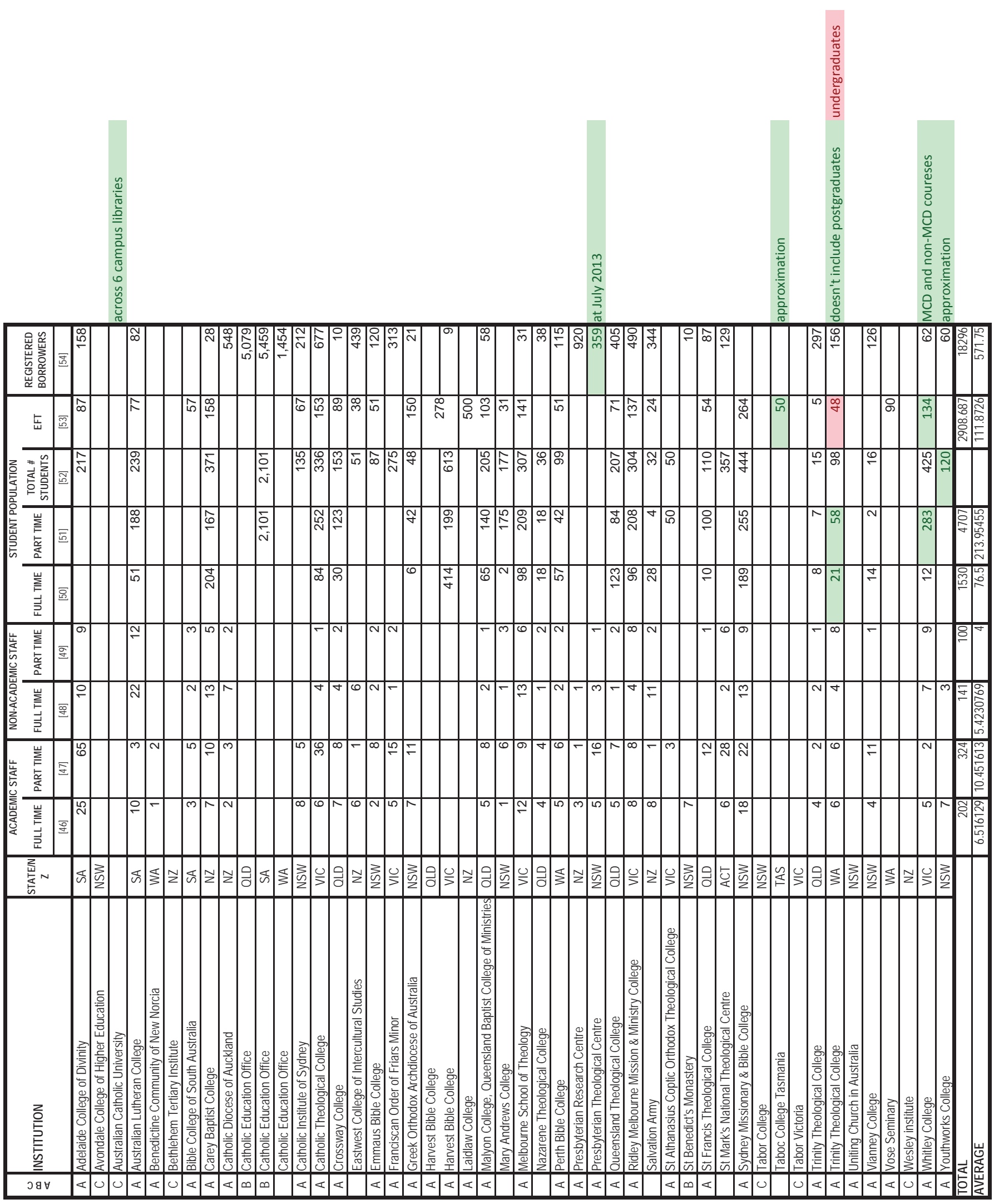


TABLE 82012 BIBUOGRAPHIC INSTRUCTION

\begin{tabular}{|c|c|c|c|c|c|}
\hline \multirow[t]{2}{*}{$\begin{array}{l}u \\
\dot{m} \\
\end{array}$} & \multirow[t]{2}{*}{ INSTITUTION } & \multirow[t]{2}{*}{$\begin{array}{l}\text { STATE } / N \\
z\end{array}$} & $\begin{array}{l}\text { BIBLIOGRAPHIC } \\
\text { \# OF ATTENDEES }\end{array}$ & $\begin{array}{l}\text { INSTRUCTION } \\
\text { \# OF SESSIONS }\end{array}$ & \\
\hline & & & [55] & [56] & \\
\hline A & Adelaide College of Divinity & SA & 25 & 4 & \\
\hline $\mathrm{C}$ & Avondale College of Higher Education & NSW & 756 & 85 & \\
\hline C & Australian Catholic University & & 19349 & 580 & across 6 campus libraries \\
\hline$A$ & Australian Lutheran College & SA & 30 & 6 & \\
\hline$A$ & Benedictine Community of New Norcia & WA & & & \\
\hline $\mathrm{C}$ & Bethlehem Tertiary Institute & NZ & & & \\
\hline$A$ & Bible College of South Australia & SA & 40 & 2 & approximation \\
\hline A & Carey Baptist College & NZ & 120 & 15 & \\
\hline$A$ & Catholic Diocese of Auckland & $\mathrm{NZ}$ & & & \\
\hline$B$ & Catholic Education Office & QLD & & & \\
\hline B & Catholic Education Office & SA & 272 & 15 & \\
\hline & Catholic Education Office & WA & & & \\
\hline A & Catholic Institute of Sydney & NSW & & 50 & \\
\hline$A$ & Catholic Theological College & $\mathrm{VIC}$ & & & \\
\hline A & Crossway College & QLD & 42 & 2 & \\
\hline & Eastwest College of Intercultural Studies & NZ & 30 & 2 & approximation \\
\hline A & Emmaus Bible College & NSW & & 4 & \\
\hline A & Franciscan Order of Friars Minor & $\mathrm{VIC}$ & 65 & 8 & \\
\hline A & Greek Orthodox Archdiocese of Australia & NSW & 8 & 2 & \\
\hline A & Harvest Bible College & QLD & & & \\
\hline$A$ & Harvest Bible College & $\mathrm{VIC}$ & & & \\
\hline$A$ & Laidlaw College & NZ & $100-150$ & $20-30$ & approximation \\
\hline$A$ & Malyon College, Queensland Baptist College of Ministries & QLD & 40 & 2 & \\
\hline & Mary Andrews College & NSW & & & \\
\hline A & Melbourne School of Theology & $\mathrm{VIC}$ & & & \\
\hline & Nazarene Theological College & QLD & 10 & 6 & \\
\hline$A$ & Perth Bible College & WA & & & \\
\hline A & Presbyterian Research Centre & $\mathrm{NZ}$ & & & \\
\hline A & Presbyterian Theological Centre & NSW & & & \\
\hline A & Queensland Theological College & QLD & 40 & 3 & \\
\hline A & Ridley Melbourne Mission \& Ministry College & $\mathrm{VIC}$ & 136 & 31 & \\
\hline & Salvation Army & NZ & 18 & 1 & \\
\hline A & St Athanasius Coptic Orthodox Theological College & $\mathrm{VIC}$ & & & \\
\hline B & St Benedict's Monastery & NSW & & & \\
\hline A & St Francis Theological College & QLD & 50 & 4 & \\
\hline & St Mark's National Theological Centre & ACT & 275 & 25 & \\
\hline A & Sydney Missionary \& Bible College & NSW & 549 & 176 & \\
\hline$C$ & Tabor College & NSW & & & \\
\hline & Taboc College Tasmania & TAS & & & \\
\hline C & Tabor Victoria & $\mathrm{VIC}$ & 65 & 24 & approximation \\
\hline$A$ & Trinity Theological College & QLD & 15 & 1 & \\
\hline$A$ & Trinity Theological College & WA & 35 & 2 & \\
\hline A & Uniting Church in Australia & NSW & & & \\
\hline A & Vianney College & NSW & & & \\
\hline$A$ & Vose Seminary & WA & & & \\
\hline $\mathrm{C}$ & Wesley institute & NZ & 250 & 9 & approximation \\
\hline$A$ & Whitley College & $\mathrm{VIC}$ & 36 & 4 & \\
\hline $\mathrm{A}$ & Youthworks College & NSW & & & \\
\hline TOT & & & 22256 & 1063 & \\
\hline AVE & RAGE & & 927.3333333 & 40.88461538 & \\
\hline
\end{tabular}

many colleges gave bibliographic instruction on an as-needed basis 
TABLE 92012 UBRARY FACIUTIES

\begin{tabular}{|c|c|c|c|c|c|c|c|c|}
\hline \multirow[t]{2}{*}{$\underset{\substack{0 \\
\&}}{0}$} & \multirow[t]{2}{*}{ INSTITUTION } & \multirow[t]{2}{*}{$\begin{array}{c}\text { STATEN } \\
Z\end{array}$} & \multirow[t]{2}{*}{$\begin{array}{l}\text { TOTAL SEATING } \\
\text { CAPACTY }\end{array}$} & \begin{tabular}{|c|} 
COMPUTERS \\
FORSTUDENTS \\
\end{tabular} & \multirow{2}{*}{\begin{tabular}{|c|} 
COMPUIERS \\
WTH INIERNEI \\
{$[59]$}
\end{tabular}} & \multirow{2}{*}{$\begin{array}{c}\begin{array}{c}\text { MRPEESS } \\
\text { AVALABEF? }\end{array} \\
{[60]} \\
\end{array}$} & \multirow{2}{*}{$\begin{array}{c}\text { COMPUIER } \\
\text { DOCKING } \\
\text { STATIONS } \\
{[61]}\end{array}$} & \\
\hline & & & & [58] & & & & \\
\hline A & Adelaide College of Divinity & SA & 40 & 8 & 12 & No & & \\
\hline $\mathrm{C}$ & Avondale College of Higher Education & NSW & 250 & 67 & 80 & Yes & & \\
\hline $\mathrm{c}$ & Australian Catholic University & & 1722 & 670 & 796 & Yes & 50 & across 6 campus libraries \\
\hline A & Australian Lutheran College & SA & 65 & 6 & 13 & Yes & 1 & \\
\hline A & Benedictine Community of New Norcia & WA & sufficient & & & No & & as submitted \\
\hline C & Bethlehem Tertiary Institute & $\mathrm{NZ}$ & 28 & 12 & 14 & Yes & & \\
\hline A & Bible College of South Australia & SA & 36 & 9 & 12 & Yes & & \\
\hline A & Carey Baptist College & $\mathrm{NZ}$ & 113 & 16 & 20 & Yes & 40 & \\
\hline A & Catholic Diocese of Auckland & $\mathrm{NZ}$ & 10 & 1 & 3 & Yes & 1 & \\
\hline$B$ & Catholic Education Office & QLD & & & & Yes & & \\
\hline \multirow{2}{*}{$B$} & Catholic Education Office & SA & 20 & 3 & 5 & Yes & & \\
\hline & Catholic Education Office & WA & 24 & 2 & 5 & Yes & & \\
\hline A & Catholic Institute of Sydney & NSW & 107 & 8 & 15 & Yes & & \\
\hline A & Catholic Theological College & $\mathrm{VIC}$ & 70 & 11 & 15 & Yes & & \\
\hline \multirow{2}{*}{ A } & Crossway College & QLD & 51 & 6 & 8 & Yes & 9 & \\
\hline & Eastwest College of Intercultural Studies & $\mathrm{NZ}$ & 35 & 4 & 6 & Yes & & \\
\hline A & Emmaus Bible College & NSW & 11 & 5 & 6 & Yes & & \\
\hline A & Franciscan Order of Friars Minor & $\mathrm{VIC}$ & 40 & 3 & 8 & Yes & & \\
\hline A & Greek Orthodox Archdiocese of Australia & NSW & 21 & 2 & 3 & No & 8 & \\
\hline A & Harvest Bible College & QLD & 5 & 3 & 4 & No & & \\
\hline A & Harvest Bible College & VIC & 10 & 8 & 9 & No & & \\
\hline A & Laidlaw College & NZ & 85 & 18 & 27 & Yes & & \\
\hline \multirow{2}{*}{ A } & Malyon College, Queensland Baptist College of Ministries & QLD & 80 & 7 & 9 & Yes & 20 & \\
\hline & Mary Andrews College & NSW & 2 & 2 & 2 & No & & \\
\hline \multirow{2}{*}{ A } & Melbourne School of Theology & $\mathrm{VIC}$ & 67 & 6 & 10 & Yes & & \\
\hline & Nazarene Theological College & QLD & 19 & 5 & 8 & Yes & 5 & \\
\hline A & Perth Bible College & WA & 30 & 5 & 9 & Yes & 13 & \\
\hline A & Presbyterian Research Centre & NZ & 58 & 4 & 4 & Yes & 2 & \\
\hline A & Presbyterian Theological Centre & NSW & 27 & 7 & 9 & Yes & 9 & \\
\hline A & Queensland Theological College & QLD & 39 & 4 & 12 & Yes & & \\
\hline \multirow{2}{*}{ A } & Ridley Melbourne Mission \& Ministry College & $\mathrm{VIC}$ & 80 & 10 & 13 & Yes & 26 & \\
\hline & Salvation Army & $\mathrm{NZ}$ & 38 & 5 & 5 & Yes & 22 & \\
\hline A & St Athanasius Coptic Orthodox Theological College & VIC & 8 & 3 & 3 & Yes & & \\
\hline$B$ & St Benedict's Monastery & NSW & 20 & & 2 & No & & \\
\hline \multirow{2}{*}{ A } & St Francis Theological College & QLD & 23 & 6 & 9 & Yes & 2 & \\
\hline & St Mark's National Theological Centre & ACT & 20 & 5 & 11 & Yes & & \\
\hline A & Sydney Missionary \& Bible College & NSW & 90 & 6 & 10 & Yes & 55 & \\
\hline \multirow[t]{2}{*}{$C$} & Tabor College & NSW & 7 & 5 & 6 & Yes & & \\
\hline & Taboc College Tasmania & TAS & & & & & & \\
\hline C & Tabor Victoria & VIC & 44 & 9 & 14 & Yes & & \\
\hline A & Trinity Theological College & QLD & 32 & 2 & 4 & Yes & & \\
\hline A & Trinity Theological College & WA & 52 & 6 & 8 & Yes & 16 & \\
\hline A & Uniting Church in Australia & NSW & 30 & 11 & 16 & Yes & 20 & \\
\hline A & Vianney College & NSW & 17 & 5 & 7 & Yes & & \\
\hline A & Vose Seminary & WA & & 9 & 12 & Yes & & \\
\hline $\mathrm{C}$ & Wesley institute & NZ & 46 & 22 & 22 & Yes & 24 & \\
\hline A & Whitley College & VIC & 45 & 12 & 14 & Yes & & \\
\hline & Youthworks College & NSW & 1 & 1 & 1 & Yes & & \\
\hline \multirow{2}{*}{\multicolumn{2}{|c|}{$\begin{array}{l}\text { TOTAL } \\
\text { AVERAGE }\end{array}$}} & & 3618 & 1019 & 1281 & & 323 & \\
\hline & & & 82.22727273 & 23.15909091 & 28.46666667 & & 17.94444444 & \\
\hline
\end{tabular}

Wireless/Wifi Available

Wireless/WifFi Not Available
40
7

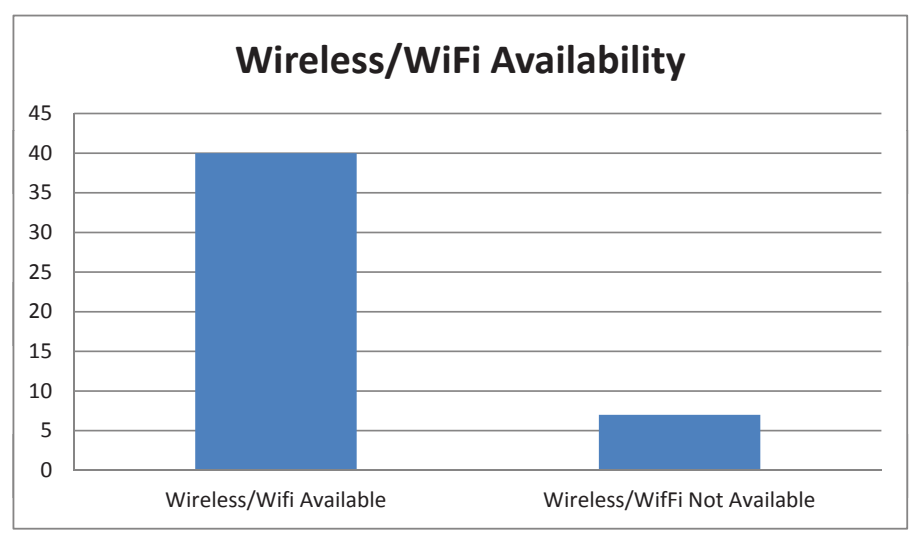


TABLE 102012 STAF SALARIES

\begin{tabular}{|c|c|c|c|}
\hline INSTITUTION & PROF. & CHURCH & OTHER \\
\hline A & 18 & 5 & 10 \\
\hline B & & & 3 \\
\hline C & & 1 & 1 \\
\hline TOTAL & 18 & 6 & 14 \\
\hline
\end{tabular}

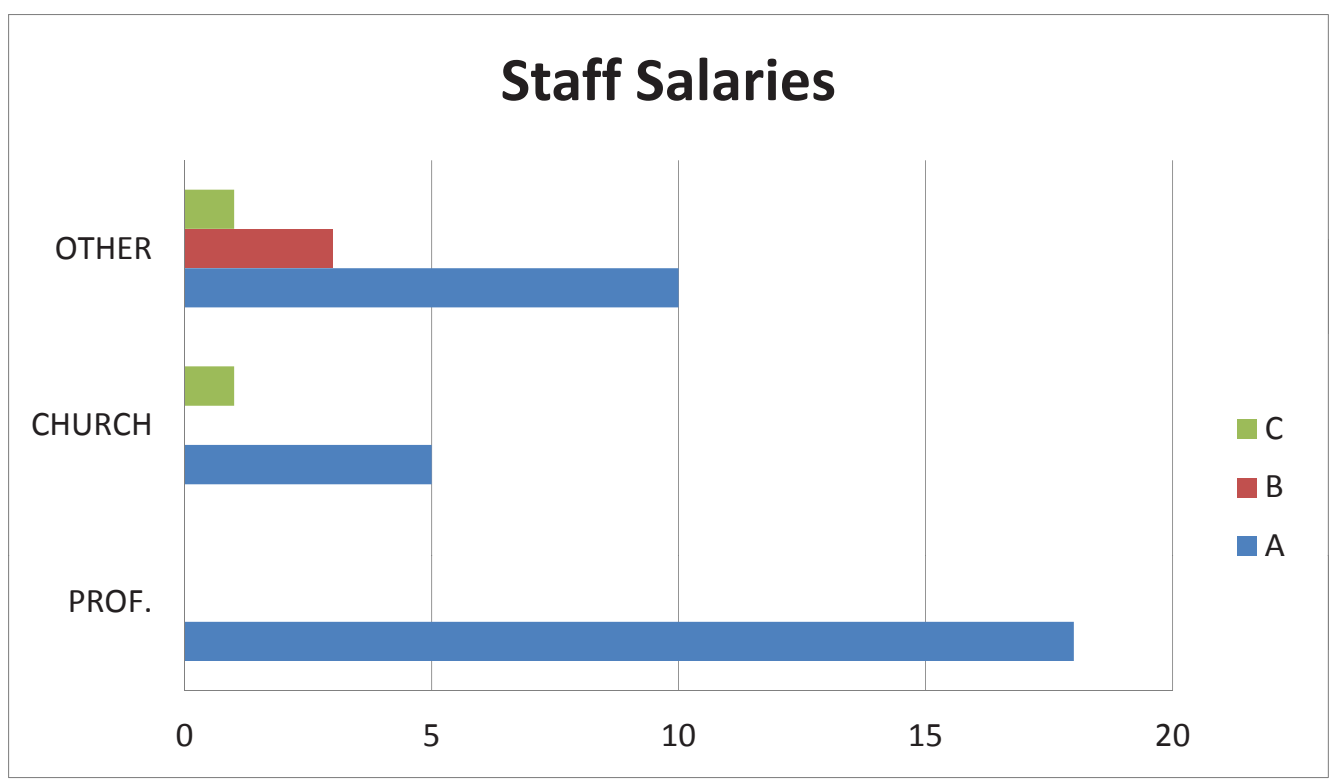


TABLE 112012 GENERAL

\begin{tabular}{|c|c|c|c|c|c|c|c|c|c|}
\hline \multirow[t]{2}{*}{ 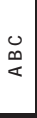 } & \multirow[t]{2}{*}{ INSTITUTION } & \multirow[t]{2}{*}{$\begin{array}{c}\text { STATE } \\
N Z\end{array}$} & \multirow[t]{2}{*}{$\begin{array}{c}\text { UBRARY SYSTEM } \\
\text { USED }\end{array}$} & ACQ. & CIRC. & SERIALS & MODULS & OPAC & OTHER \\
\hline & & & & \multicolumn{6}{|c|}{ [64] } \\
\hline A & Adelaide College of Divinity & SA & Voyager & & Yes & & Yes & Yes & Reports \\
\hline $\mathrm{C}$ & Avondale College of Higher Education & NSW & Aleph & Yes & Yes & Yes & Yes & Yes & IILL \\
\hline $\mathrm{C}$ & Australian Catholic University & & Aleph & Yes & Yes & Yes & Yes & Yes & \\
\hline A & Australian Lutheran College & SA & SirsiDynix & & Yes & & Yes & Yes & \\
\hline A & Benedictine Community of New Norcia & WA & Inmagic DB & & & Yes & Yes & & \\
\hline $\mathrm{C}$ & Bethlehem Tertiary Institute & $\mathrm{NZ}$ & Oliver & Yes & Yes & Yes & Yes & Yes & \\
\hline A & Bible College of South Australia & SA & Alice & Yes & Yes & & Yes & Yes & Management-Stocktake \\
\hline A & Carey Baptist College & $\mathrm{NZ}$ & Liberty & Yes & Yes & Yes & Yes & Yes & \\
\hline A & Catholic Diocese of Auckland & $\mathrm{NZ}$ & Liberty & Yes & Yes & Yes & Yes & Yes & \\
\hline B & Catholic Education Office & QLD & Destiny & Yes & Yes & Yes & Yes & Yes & \\
\hline \multirow[t]{2}{*}{$\mathrm{B}$} & Catholic Education Office & SA & AIMSMasterfile & & Yes & & Yes & Yes & \\
\hline & Catholic Education Office & WA & eLibrary & & Yes & & Yes & Yes & \\
\hline A & Catholic Institute of Sydney & NSW & Aleph & Yes & Yes & Yes & Yes & Yes & \\
\hline A & Catholic Theological College & $\mathrm{VIC}$ & & & Yes & & Yes & Yes & \\
\hline \multirow{2}{*}{ A } & Crossway College & QLD & Destiny & Yes & Yes & Yes & Yes & Yes & \\
\hline & Eastwest College of Intercultural Studies & $\mathrm{NZ}$ & Acces-It Library & Yes & Yes & Yes & Yes & Yes & \\
\hline A & Emmaus Bible College & NSW & $\mathrm{KOHA}$ & Yes & Yes & Yes & Yes & Yes & \\
\hline A & Franciscan Order of Friars Minor & $\mathrm{VIC}$ & Liberty & Yes & Yes & Yes & Yes & Yes & \\
\hline A & Greek Orthodox Archdiocese of Australia & NSW & Liberty & Yes & Yes & & Yes & Yes & System \\
\hline$A$ & Harvest Bible College & QLD & Destiny & & Yes & Yes & Yes & Yes & \\
\hline A & Harvest Bible College & VIC & Alice & Yes & Yes & Yes & Yes & Yes & \\
\hline A & Laidlaw College & NZ & Liberty & Yes & Yes & Yes & Yes & Yes & ILL, Federated search \\
\hline \multirow{2}{*}{ A } & Malyon College, Queensland Baptist College of & QLD & Liberty & Yes & Yes & Yes & Yes & Yes & IILL \\
\hline & Mary Andrews College & NSW & Filemaker Pro & & & & & & \\
\hline \multirow{2}{*}{ A } & Melbourne School of Theology & $\mathrm{VIC}$ & Liberty & & Yes & Yes & Yes & Yes & \\
\hline & Nazarene Theological College & QLD & Destiny & Yes & Yes & Yes & Yes & & \\
\hline A & Perth Bible College & WA & $\mathrm{KOHA}$ & & Yes & & Yes & & \\
\hline A & Presbyterian Research Centre & $\mathrm{NZ}$ & Voyager & & & & Yes & Yes & \\
\hline A & Presbyterian Theological Centre & NSW & Accesslt & Yes & Yes & & Yes & Yes & \\
\hline A & Queensland Theological College & QLD & Liberty & & Yes & & Yes & Yes & Self-circulation \\
\hline \multirow[t]{2}{*}{ A } & Ridley Melbourne Mission \& Ministry College & VIC & Horizon & & Yes & & Yes & Yes & \\
\hline & Salvation Army & $\mathrm{NZ}$ &. $\mathrm{eLm}$ & Yes & Yes & & Yes & Yes & \\
\hline$A$ & St Athanasius Coptic Orthodox Theological College & $\mathrm{VIC}$ & BiblioTech & & & & & Yes & \\
\hline$B$ & St Benedict's Monastery & NSW & Library World & & Yes & & Yes & Yes & Reports \\
\hline \multirow[t]{2}{*}{ A } & St Francis Theological College & QLD & Liberty & Yes & Yes & Yes & Yes & Yes & ILL \\
\hline & St Mark's National Theological Centre & ACT & Unilinc Aleph & Yes & Yes & Yes & Yes & Yes & \\
\hline A & Sydney Missionary \& Bible College & NSW & Athena & Yes & Yes & Yes & Yes & Yes & \\
\hline \multirow[t]{2}{*}{$\mathrm{C}$} & Tabor College & NSW & Unilinc Aleph & & Yes & & Yes & Yes & \\
\hline & Taboc College Tasmania & TAS & Bookmark & & & & & & \\
\hline C & Tabor Victoria & VIC & Destiny & & Yes & & Yes & Yes & \\
\hline A & Trinity Theological College & QLD & Liberty & Yes & Yes & Yes & Yes & Yes & ILL; Z39.50; Syndetics \\
\hline A & Trinity Theological College & WA & OCLCWorldshare & Yes & Yes & Yes & Yes & Yes & Discovery layer \\
\hline A & Uniting Church in Australia & NSW & Liberty & Yes & Yes & Yes & Yes & Yes & \\
\hline A & Vianney College & NSW & Destiny & & Yes & & Yes & Yes & \\
\hline $\mathrm{A}$ & Vose Seminary & WA & Liberty & & & & & & \\
\hline$C$ & Wesley institute & $\mathrm{NZ}$ & Liberty & & & & & & \\
\hline$A$ & Whitley College & $\mathrm{VIC}$ & Liberty & Yes & Yes & Yes & Yes & Yes & \\
\hline A & Youthworks College & NSW & Library World & & & & & & \\
\hline
\end{tabular}




\section{Library Systems Used}

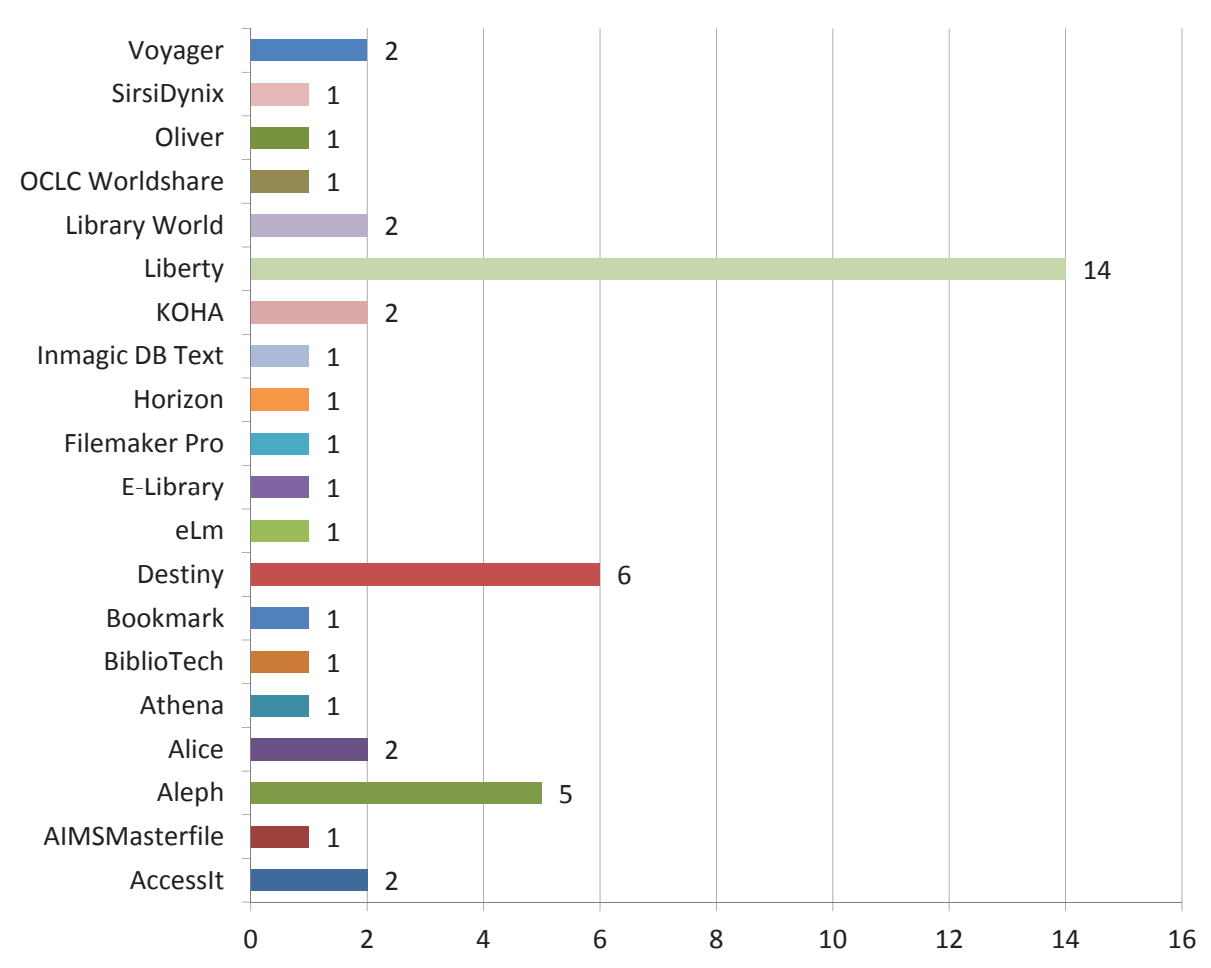

$\begin{array}{lc}\text { Accesslt } & 2 \\ \text { AlMSMasterfile } & 1 \\ \text { Aleph } & 5 \\ \text { Alice } & 2 \\ \text { Athena } & 1 \\ \text { BiblioTech } & 1 \\ \text { Bookmark } & 1 \\ \text { Destiny } & 6 \\ \text { eLm } & 1 \\ \text { E-Library } & 1 \\ \text { Filemaker Pro } & 1 \\ \text { Horizon } & 1 \\ \text { Inmagic DB Text } & 1 \\ \text { KOHA } & 2 \\ \text { Liberty } & 14 \\ \text { Library World } & 2 \\ \text { OCLC Worldshare } & 1 \\ \text { Oliver } & 1 \\ \text { SirsiDynix } & 1 \\ \text { Voyager } & 2\end{array}$

